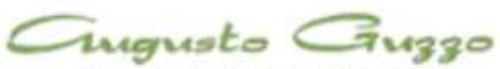

REVISTA ACADEMICA

\title{
Introdução À Mecânica Quântica Não-Comutativa: Situação Relativística E Não-Relativística
}

\section{Introduction To Noncommutative Quantum Mechanics: Relativistic And Nonrelativistic Situation}

\author{
Carlos Alberto Stechhahn da Silva
}

Carlos Alberto Stechhahn da Silva é doutor em física e professor da Faculdades Integradas Campos Salles.e-mail:stecphysics@gmail.com

\section{Resumo}

Neste trabalho são apresentadas algumas das motivações para o estudo da mecânica quântica nãocomutativa na situação relativística e não-relativística. Após a introdução do produto não-comutativo para as funções no espaço de fase e do produto Moyal em suas diferentes formas expressamos, na situação relativística e com o uso da equação de Dirac, como se transforma o tensor do campo eletromagnético no plano não-comutativo. Aplicamos tais estudos, na condição não-relativística, ao oscilador harmônico não-comutativo 2-D usando o método dos operadores de Dirac. Constatamos que o espectro da energia é degenerado e sua degenerescência é levantada pela não-comutatividade.

Palavras-chave: Mecânica Quântica, não-comutatividade, não-comutativo, tensor do campo eletromagnético e oscilador harmônico.

\begin{abstract}
This paper presents some of the motivations for the study of the noncommutative quantum mechanics in both relativistic and nonrelativistic situation. After the introduction of noncommutative product for the functions in space phase and Moyal product in its different forms we express in relativistic situation and using Dirac equation how the electromagnetic tensor field changes in a noncommutative plane. We apply such studies in a nonrelativistic condition to the 2-D noncommutative harmonic oscillator using the method of Dirac operators. We observe that the energy spectrum is degenerate and its degeneracy is lifted by the noncommutativity.
\end{abstract}

Keywords: Quantum mechanics, noncommutativity, noncommutative, electromagnetic tensor field and harmonic oscillator.

202 SILVA, C.A.S.: Introdução A Mecânica Quântica Não-Comutativa: Situação Relativística E NãoRelativística. 


\section{Introdução}

A humanidade pode experimentar o vislumbrar e o fascínio de duas teorias que revolucionaram a física no século XX e nossa visão de mundo. De um lado a mecânica quântica (GRIFFITHS, 2005), MQ, dedicada ao estudo de fenômenos microscópicos (moléculas, átomos e partículas elementares) e, de outro, a Relatividade Restrita de Einstein, $R R$, (RINDLER, 2006) descrevendo os chamados fenômenos relativísticos, ou seja, de objetos que se movem com a velocidade próxima da velocidade da luz; esta teoria uniu ainda os conceitos de espaço e tempo (espaço-tempo) no mesmo patamar de igualdade. Computadores, celulares, aparelhos de ressonância magnética e cirurgias com o uso de "lasers", entre outros, são exemplos do emprego dessas duas teorias físicas. Não deixou de ter validade a física clássica de Newton, bem como o eletromagnetismo de Maxwell, não obstante, uma série de experimentos, alguns dos quais notadamente no século XIX, não podiam ser por elas explicados.

A união dessas duas teorias, a $M Q$ e a $R R$, deram origem à teoria quântica de campos, TQC, que estuda o comportamento das partículas elementares (elétrons, quarks, neutrinos, etc.) como modos de vibração de campos fundamentais que preenchem todo o espaço-tempo. A TQC surgiu na década de 1920 e um dos resultados desses estudos foi o surgimento dos fótons (os quanta do campo eletromagnético) para explicar o espectro de radiação do corpo negro. Particularmente, quando a teoria quântica de campos descreve partículas carregadas (campos de matéria) interagindo com campos eletromagnéticos (fótons) temos a eletrodinâmica quântica (BASSALO, 2006). Um de seus resultados teóricos (correções para o fator giromagnético, g) se encontram numa concordância com o experimental em dez casas decimais. Esta é a mais precisa teoria científica que se tem conhecimento, uma das concordâncias teóricoexperimental mais notáveis que o ser humano já produziu. Os fundamentos da TQC foram estabelecidos por Dirac em 1927. Hoje, área ainda de vasta pesquisa, é uma disciplina dos cursos de pós-graduação em física. Uma proposta para o ensino de teoria quântica de campos na graduação pode ser encontrada em (OLIVEIRA, 2011).

Vale ressaltar, no entanto que, um de seus pilares, a $M Q$, tem no princípio da incerteza de Heisenberg um dos fundamentos que a norteiam. Por este princípio, contrariamente ao que prevê a mecânica clássica, não se pode medir, por exemplo, a posição e o momento (massa vezes velocidade) de um elétron com precisão infinita. Neste caso, $x \cdot p_{x} \neq p_{x} . x$. Ela é, portanto, constituída de uma álgebra de operadores que não comutam. Mas, em $M Q$ ainda é possível medirmos dois observáveis 
posição, $x$ e $y$, com precisão ilimitada, nesse caso dizemos que $x$ e $y$ comutam, i.e., $x \cdot y-y \cdot x=$ $[x, y]=0 . \quad \mathrm{E}$ tomando como base esse princípio, bem como seus diversos postulados, é que se estrutura a mecânica quântica, aqui chamada de comutativa.

Contudo, recentemente, tem havido um grande interesse no estudo de teorias no espaço não-comutativo, $N C$. Neste contexto, além das relações de Heisenberg serem válidas, são estudados modelos em que as coordenadas do espaço-tempo não comutam.

Em Física a ideia da não-comutatividade do espaço tornou-se muito popular nos últimos anos como uma descrição mais fundamental do espaço-tempo na escala de Planck (regime das flutuações quânticas, i.e., distâncias da ordem de $\ell_{p}=\left(\frac{G \hbar}{c^{3}}\right)^{1 / 2} \sim 10^{-33} \mathrm{~cm}, \quad$ ou ainda, alternativamente, como um limite particular da teoria quântica da gravitação. Estudos recentes de teorias de cordas (SEIBERG;WITTEN,1999) revelaram que a dinâmica de uma corda aberta na presença de um campo magnético de fundo pode ser descrita por uma teoria de calibre (gauge) deformada pelo produto Moyal. Algumas características e propriedades deste produto serão estudadas neste trabalho. Este fato contribuiu para os estudos de teorias quânticas de campos definidas num espaço não-comutativo.

A proposta inicial da nãocomutatividade das coordenadas do espaçotempo, envolta no formalismo da TQC, é, no entanto, bastante antiga. Segundo a literatura, tem sua origem datada de 1947, embora sugestões anteriores nesse sentido já houvessem ocorrido. A ideia, contudo, foi abandonada após o grande sucesso do programa de renormalização (solução do problema dos infinitos em cálculos perturbativos). A literatura envolvendo este tema é bastante vasta e uma revisão pode ser encontrada em (GIROTTI, 2003).

Neste cenário, cientes da grande precisão da TQC no estudo de física em alta energia, podemos estudar muitos fenômenos com o uso da mecânica quântica.

\section{Mecânica Quântica Não- Relativística}

No final do século XIX a grande maioria dos físicos acreditava que os fenômenos da natureza se encontravam completamente descritos pela ciência e que apenas alguns detalhes precisavam ser trabalhados. A física clássica de Newton, juntamente com o eletromagnetismo de Maxwell, era capaz de dar conta da descrição dos sistemas macroscópicos incluindo o cosmos. Contudo, assim que novas técnicas experimentais foram sendo desenvolvidas, e adentraram ao reino do sistema atômico, surgiram situações e fenômenos que não podiam ser resolvidos ou descritos pela física clássica. A radiação de corpo negro, a estabilidade da órbita do elétron, o espectro atômico, a foto emissão de elétrons, entre 
outros, foram os experimentos iniciais que serviram de base para a formação da mecânica quântica. Cabe aqui ressaltar que os resultados e estudos da $M Q$ fogem do nosso dia-a-dia macroscópico; seus resultados experimentais estão fora do alcance e percepção humanos.

\subsection{Base conceitual e características}

$\mathrm{Na}$ física de Newton (GOLDSTEIN, 1980) o conhecimento pleno da posição e velocidade de uma partícula pode fornecer uma descrição completa do sistema. $\mathrm{Na}$ mecânica quântica, por outro lado, a descrição de um sistema é fornecida por uma função, chamada função estado do sistema, a qual não pode ser diretamente observada. A informação dada pela função estado é estatística ou probabilística. Outros ingredientes, tais como, observáveis (energia, momento, momento angular e outras variáveis dinâmicas), representados por operadores, estes, objetos matemáticos abstratos, fazem parte dos sistemas quânticos. Nestes, ocorrem ainda uma interação entre o aparato de medida e o sistema que está sendo observado. No nível clássico tal interação pode ser suposta infinitamente pequena. No nível quântico, contudo, ela não pode ser diminuída deliberadamente. A observação de uma propriedade A (energia, por exemplo) gera uma mudança imprevisível em um algum outro observável B (o tempo, neste caso). Esta é uma característica do processo de medida em sistemas quânticos. Tais perturbações ou incertezas mútuas não se deve a técnica experimental em uso; elas são uma consequência da medida. A natureza quântica opera dessa forma. A existência desse efeito em pares de variáveis complementares foi formulada inicialmente por Heisenberg e é conhecida como "princípio da incerteza". Outro aspecto, não menos importante, é o aspecto ondulatório das partículas. O espalhamento de um feixe de elétrons por um cristal foi feito inicialmente por Davisson e Germer em 1927. Tal experimento constatou a previsão do físico francês Louis de Broglie na qual, apoiado no princípio de Fermat da óptica e no princípio da ação mínima da mecânica, postulou que, assim como a luz (radiação) possuía um caráter corpuscular, a matéria (elétrons) deveria apresentar um caráter ondulatório. Assim, ele associou ao momento $p$ da partícula um comprimento de onda $\lambda$ dado por:

$$
\lambda=\frac{h}{p}
$$

onde $h$ é a constante de Planck. Esta hipótese também está em conflito com a física clássica. Em $M Q$, ambas, partículas e ondas, tem uma natureza dual, i.e., estão associadas a uma mesma entidade. Isto implica que os elétrons podem assumir um caráter de partícula ou de onda dependendo do experimento. Há ainda outras situações físicas na $M Q$ que divergem da teoria clássica, tais como, transmissão de partículas num potencial degrau numa condição em que para a física de Newton todas as partículas deveriam ser refletidas, interpretação probabilística para a posição dos elétrons, efeito 
túnel, fotoelétrico, Compton, entre outros (GASIOROWICZ, 2003).

\subsection{Parênteses de Poisson e os esquemas} de quantização

Quando idealizamos um sistema dinâmico clássico, com um número finito de graus de liberdade, pensamos em algo como um sistema de $n$-partículas em que estas são especificadas em qualquer tempo por suas posições e momentos instantâneos (STECHHAHN, 2005). O estado do sistema é completamente especificado por um ponto no espaço de fase $M 2 n$-dimensional. $M$ é uma variedade, e em coordenadas canônicas um ponto $\times$ em $M$ é escrito como

$$
\begin{aligned}
& x=(q, p) \\
& =\left(q_{1}, \ldots, q_{n}, p_{1}, \ldots, p_{n}\right)
\end{aligned}
$$

A energia do sistema é obtida calculando a função de Hamilton no ponto do espaço de fase $x_{0}=H\left(q_{0}, p_{0}\right)$ que caracteriza o estado do sistema neste tempo, ou seja, $E=H\left(q_{0}, p_{0}\right)$. Em geral, funções sobre uma variedade são multiplicadas entre si de modo pontual, i.e., dadas duas funções $f$ e $g$ seu produto $f g$ é uma função

$$
(f g)(x)=f(x) g(x)
$$

Assim, no contexto da Mecânica Clássica os observáveis constroem uma álgebra comutativa chamada álgebra clássica comutativa dos observáveis.
$\mathrm{Na}$ mecânica Hamiltoniana, se considerarmos $f(q, p, t)$ uma variável dinâmica arbitrária, i.e, uma função qualquer das variáveis canônicas e do tempo, teremos, usando as equações de Hamilton:

$$
\begin{gathered}
\frac{d f}{d t}=\sum_{k=1}^{n}\left(\frac{\partial f}{\partial q_{k}} \frac{\partial H}{\partial p_{k}}-\frac{\partial f}{\partial p_{k}} \frac{\partial H}{\partial q_{k}}\right) \\
+\frac{\partial f}{\partial t}
\end{gathered}
$$

O colchete ou parêntese de Poisson

$$
\begin{aligned}
\{f, g\}=\sum_{i=1}^{n}( & \frac{\partial f}{\partial q_{i}} \frac{\partial g}{\partial p_{i}} \\
& \left.-\frac{\partial f}{\partial p_{i}} \frac{\partial g}{\partial q_{i}}\right)
\end{aligned}
$$

pode ser escrito numa forma compacta usando a notação:

$$
f \overleftarrow{\partial}_{q_{i}} g=\frac{\partial f}{\partial q_{i}} g
$$

e

$$
f \vec{\partial}_{p_{i}} g=f \frac{\partial g}{\partial p_{i}}
$$

E a equação (5) torna-se:

$$
\{f, g\}=f\left(\overleftarrow{\partial}_{q_{i}} \vec{\partial}_{p_{i}}-\vec{\partial}_{p_{i}} \overleftarrow{\partial}_{q_{i}}\right) g
$$

onde usamos a convenção de Einstein sobre índices repetidos. A equação ( 6 ) pode ser ainda mais abreviada usando $x$ para representar ponto do espaço de fase $x=\left(x_{1}, \ldots, x_{2 n}\right)$ e o tensor de Poisson $\alpha^{i j}$, onde os índices $i$ e $j$ percorrem de 1 a $2 n$. Em coordenadas canônicas $\alpha^{i j}$ é representado pela matriz 


$$
\alpha=\left(\begin{array}{cc}
0 & I_{n} \\
-I_{n} & 0
\end{array}\right)
$$

onde $I_{n}$ é a matriz identidade $n \times n$. Assim, a Eq. ( 5 ) terá a seguinte forma:

$$
\{f, g\}(x)=\alpha^{i j} \partial_{i} f(x) \partial_{j} g(x)
$$

$\operatorname{com} \partial_{i}=\partial / \partial x_{i}$

A evolução de um sistema dinâmico clássico é dada pelas equações de Hamilton, as quais, escritas em termos dos parênteses de Poisson, tornam-se:

$$
\begin{gathered}
\dot{q}_{i}=\frac{\partial H}{\partial p_{i}}=\left\{q_{i}, H\right\} \\
\dot{p}_{i}=-\frac{\partial H}{\partial q_{i}}=\left\{p_{i}, H\right\}
\end{gathered}
$$

No entanto, em sistemas quânticos a evolução temporal da função de onda é dada pela equação de Schrödinger

$$
i \hbar \frac{\partial \psi}{\partial t}=-\frac{\hbar^{2}}{2 m} \frac{\partial^{2} \psi}{\partial x^{2}}+V(x, t) \psi
$$

Veremos a seguir como a mecânica quântica NC é implementada (FERRARI, 2010), o produto Moyal não-comutativo, a nova álgebra de Heisenberg para os operadores posição, e como a própria equação de Schrödinger se transforma face a mudança $V(x, t) \psi \rightarrow V(x, t) \star \psi$.

\subsection{A Mecânica Quântica NC e o} Produto Estrela

A não-comutatividade na $M Q$ surge com a introdução do produto não-comutativo para as funções no espaço de fase. Os observáveis são representados por operadores no espaço de Hilbert que não comutam e as quantidades físicas são os autovalores desses operadores. Vamos considerar funções a valores complexos sobre uma variedade de Poisson em diferentes esquemas de quantização. $O$ produto estrela $(f * g)$ é uma nova função descrita por uma série infinita:

$$
\begin{aligned}
(f * g)=f g & +(i h) C_{1}(f, g)+\mathcal{O}\left(\hbar^{2}\right) \\
& =\sum_{n=0}^{\infty}(i \hbar)^{n} C_{n}(f, g)
\end{aligned}
$$

O primeiro termo da série é o produto

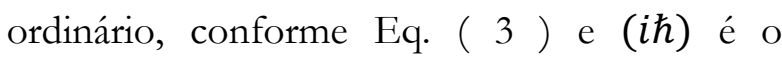
parâmetro de deformação, o qual varia continuamente. Os coeficientes $C_{n}$ serão tais que teremos uma álgebra NC formada a partir de funções com esta nova lei de multiplicação, ou seja, uma deformação da álgebra comutativa original a qual usa multiplicação pontual de funções. Os coeficientes $C_{n}(f, g)$ são funções constituídas de derivadas das funções $f$ e $g$ e possuem as seguintes propriedades:

I. Associatividade: 


$$
\begin{aligned}
& \sum_{j+k=n} C_{j}\left(C_{k}(f, g), h\right) \\
= & \sum_{j+k=n} C_{j}\left(f, C_{k}(g, h)\right)
\end{aligned}
$$

Esta propriedade mostra que o produto estrela, ou produto Moyal, é associativo, i.e.,

$$
(f * g) * h=f *(g * h)
$$

II. Correspondência clássica:

$$
C_{0}(f, g)=f g
$$

Ou seja, no $\lim _{\hbar \rightarrow 0} f * g=f g$.
III. Conexão entre os comportamentos clássicos e quânticos:

IV.

$$
C_{1}(f, g)=C_{1}(g, f)=\{f, g\}
$$

Escolhendo $C_{1}(f, g)$ antissimétrico, $C_{1}(f, g)=$ $-C_{1}(g, f)$, temos:

$$
C_{1}(f, g)=\frac{1}{2} \alpha^{i j} \partial_{i} f(x) \partial_{j} g(x) \underset{(6)}{=} \frac{1}{2}\{f, g\}
$$

Definindo o comutador estrela por este novo produto:

$$
[f, g]_{*}=f * g-g * f
$$

Notando que cada produto, até a primeira ordem

$$
\begin{aligned}
& \text { em } \hbar \text {, é: } \\
& f * g=f g+(i \hbar) C_{1}(f, g) \\
& \text { e } \\
& g * h=f g+(i \hbar) C_{1}(g, f) .
\end{aligned}
$$

Subtraindo a Eq. (13) da Eq. (14) teremos o princípio da correspondência:

$$
\begin{aligned}
& \lim _{\hbar \rightarrow 0} \frac{1}{i \hbar}=[f, g]_{*} \\
& =\{f, g\} .
\end{aligned}
$$

Para espaços euclidianos planos, $M=$ $\mathbb{R}^{2 n}$, as componentes do tensor de Poisson $\alpha^{i j}$ podem ser tomadas como constantes.

\subsection{O Produto Moyal e suas formas}

Os coeficientes de ordem mais alta podem ser obtidos a partir da exponenciação de $C_{1}$. Com este procedimento obteremos o produto Moyal

$$
\begin{aligned}
& f *_{M} g \\
& =f \exp \left[\left(\frac{i \hbar}{2}\right) \alpha^{i j} \overleftarrow{\partial}_{i} \vec{\partial}_{j}\right] g
\end{aligned}
$$

$\operatorname{com} \alpha^{i j}$ o tensor anti-simétrico de Poisson.

1.4.1 Em termos das coordenadas canônicas

$\mathrm{Na}$ física clássica $p$ e $q$ são variáveis dinâmicas fundamentais (coordenadas canônicas) e o produto Moyal em termos dessas coordenadas pode ser escrito como: 


$$
\begin{aligned}
f *_{M} g(q, p)= & f(q, p) \exp \left[( \frac { i \hbar } { 2 } ) \left(\overleftarrow{\partial}_{q} \vec{\partial}_{p}\right.\right. \\
& \left.\left.-\overleftarrow{\partial}_{p} \vec{\partial}_{q}\right)\right] g(q, p)=
\end{aligned}
$$

$=\sum_{m, n=0}^{\infty}\left(\frac{i \hbar}{2}\right)^{m+n} \frac{(-1)^{m}}{m ! n !}\left(\partial_{p}^{m} \partial_{q}^{n} f\right)\left(\partial_{p}^{n} \partial_{q}^{m} g\right)$

\subsection{2 "Shift Fórmula"}

Uma outra forma bastante útil nos cálculos é aquela na qual as coordenadas e momentos estão transladados de uma quantidade relacionada a derivadas dessas quantidades:

$$
\begin{aligned}
& f *_{M} g(q, p) \\
& =f\left(q+\frac{i \hbar}{2} \vec{\partial}_{p}, p\right. \\
& \left.-\frac{i \hbar}{2} \vec{\partial}_{q}\right) g(q, p)
\end{aligned}
$$

A prova da expressão acima pode ser obtida expandindo a soma que há na primeira das equações em ( 17 ) que irá coincidir com a expansão em série de Taylor do lado direito da Eq. ( 18 ). Tais expressões se identificam no seguinte resultado:

$$
\begin{gathered}
f *_{M} g(q, p)=f g+\frac{i \hbar}{2}\left(\partial_{q} f \partial_{p} g-\partial_{p} f \partial_{q} g\right) \\
+ \\
+\left(\frac{i \hbar}{2}\right)^{2}\left[\partial_{q}^{2} f \partial_{p}^{2} g-2 \partial_{p} \partial_{q} f \partial_{q} \partial_{p} g\right. \\
\left.+\partial_{p}^{2} f \partial_{q}^{2} g\right]+\cdots
\end{gathered}
$$

Todo esse ferramental físico e matemático da mecânica quântica nãocomutativa pode ser resumido numa sequência ou procedimentos usualmente empregados na área. Espaços não-comutativos são caracterizados por operadores posição $\hat{x}_{\mu}$ satisfazendo a relação

$$
\begin{aligned}
& {\left[\hat{x}_{\mu}, \hat{x}_{\nu}\right]} \\
& =i \theta_{\mu \nu}
\end{aligned}
$$

onde $\theta_{\mu \nu}$ é uma matriz constante e antissimétrica com dimensão de comprimento ao quadrado.

Inicialmente, se escreve a equação de Schrödinger para um potencial $V(x)$ que se quer estudar. A forma do potencial é de livre escolha bem como a dimensão do espaço (1 a Ndimensões). A seguir, se impõe que todo o sistema está inserido num espaço $N C$, ou seja, as variáveis dinâmicas, $x_{i}$ e $p_{i}$ (operadores) serão identificadas por um chapéu ("batted" quantidades)

$$
\begin{gathered}
\hat{x}_{j}=x_{j}-\frac{1}{2 \hbar} \sum_{k} \theta_{j k} p_{k} \\
\hat{p}_{j}=p_{j}
\end{gathered}
$$

e teremos essas novas variáveis $x_{j}$ e $p_{j}$ satisfazendo a álgebra de Heisenberg usual da $M Q$

$$
\begin{gathered}
{\left[x_{j}, x_{j}\right]=\left[p_{i}, p_{j}\right]=0} \\
{\left[x_{i}, p_{j}\right]=i \hbar \delta_{i j}}
\end{gathered}
$$


Com tais modificações a equação de Schrödinger voltará a ter sua forma padrão agora com o potencial modificado

$$
\begin{aligned}
& V(x, t) \psi \rightarrow V(x, t) \star \psi \\
& =V\left(x_{j}-\frac{1}{2 \hbar} \sum_{k} \theta^{j k} p_{k}\right) .
\end{aligned}
$$

Uma discussão muito proeminente no espaço-tempo $N C$ é a violação da unitariedade e causalidade quando $\theta^{0 i} \neq 0$. Aqui, por simplicidade, iremos considerar uma forma particular da matriz $\theta^{i j}$ em que a nãocomutatividade é diferente de zero somente num plano espacial particular, i.e., $\theta^{i 0}=0$.

\section{Mecânica Quântica Relativística}

A mecânica quântica não-relativística é regida pela equação de Schrödinger, conforme Eq. ( 8 ), cuja solução, a função de onda $\psi$ tem uma interpretação física em que $\rho=|\psi|^{2}$ representa a densidade de probabilidade. Ou seja, informa onde é mais provável de se encontrar a partícula quântica que se está estudando, neste caso não-relativística e sem spin.

Por meio da equação da continuidade

$$
\frac{\partial \rho}{\partial t}+\nabla \cdot \vec{\jmath}=0
$$

temos a conservação de probabilidade; a probabilidade de se encontrar a partícula em algum lugar é 1 e esta propriedade independe do tempo. A equação de continuidade é obtida a partir da equação de Schrödinger, no entanto, ela não é compatível com a relatividade restrita. Se considerarmos as transformações de Lorentz

$$
\begin{aligned}
& x^{\prime}=\gamma(x-v t) \\
& t^{\prime}=\gamma\left(t-\frac{v x}{c^{2}}\right)
\end{aligned}
$$

e escrevermos a equação de Schrödinger em termos de $x^{\prime}$ e $t^{\prime}$ a equação mudará de forma. Todavia, a equação de Schrödinger não pode ser diferente em referenciais diferentes, dado que observadores nesses locais devem ver a mesma física. Dessa forma, a mudança $x \rightarrow x^{\prime}$ e $t \rightarrow t^{\prime}$ mostra que a equação de Schrödinger não é relativística, i.e., invariante pela RR. A equação de Schrödinger, portanto, não é a equação dinâmica apropriada para descrever partículas relativísticas. Para obtermos uma equação de onda relativística devemos começar considerando partículas livres com a relação relativística (GREINER, 2000)

$$
p^{\mu} p_{\mu}=\frac{E^{2}}{c^{2}}-\boldsymbol{p} \cdot \boldsymbol{p}=m_{0}^{2} c^{2} .
$$

Imponto que o operador $p^{\mu} \rightarrow i \hbar\{\partial /$ $\partial(c t)-\nabla\}$ obtemos a equação de KleinGordon, $K G$, para partículas livres

$$
\left(\square+\frac{m_{0}^{2} c^{2}}{\hbar^{2}}\right) \psi=0
$$


Nesta equação se verifica a covariância de Lorentz, no entanto, sua densidade de probabilidade não é definida positiva, i.e., não pode ser tomada como tal. Isto ocorre porque a equação de $K G$ é de segunda ordem no tempo.

\subsection{Partículas relativísticas de spin 1/2.}

Paul Adrien Maurice Dirac, após a publicação de seu artigo sobre a teoria quântica da radiação em 1927 passou a construir a teoria relativística para os elétrons. Observou que a não-relativística equação de Schrödinger não possuía probabilidades negativas porque ela era linear no tempo. No entanto, a equação de Klein-Gordon era quadrática no tempo e possuía probabilidades negativas. Dessa forma, Dirac tentou escrever uma equação de onda que fosse linear no tempo e que estivesse vinculada à equação ( 25 ) da energia relativística. A ideia original foi obter a raiz quadrada da equação da energia. Iniciando com uma equação de primeira ordem no tempo e no espaço $(\hbar=c=1)$ :

$$
i \frac{\partial \psi}{\partial t}=\left(-i \alpha_{i} \nabla^{i}+\beta m\right) \psi
$$

onde $\alpha_{i}$ e $\beta$ agora são matrizes as quais atuam numa matriz coluna $\psi$ a qual adquire um caráter espinorial (GOMES, 2002:30). Elevando-se ao quadrado o lado direito da expressão acima deseja-se recuperar a equação da energia relativística e se impõe que: $-\frac{\partial^{2} \psi}{\partial t^{2}}=(-i \vec{\alpha} \cdot \vec{\nabla}+\beta m)^{2} \psi=\left(-\nabla^{2}+\right.$ $\left.m^{2}\right) \psi(28)$

Isto será perfeitamente possível se nós exigirmos que as matrizes obedeçam as seguintes relações:

$$
\begin{gathered}
\left\{\alpha_{i}, \alpha_{j}\right\}=2 \delta_{i j} \\
\left\{\alpha_{i}, \beta\right\}=0 \\
\alpha_{i}^{2}=\beta^{2}=1
\end{gathered}
$$

As matrizes $\alpha_{i}$ e $\beta$ são de ordem $4 \times 4$ e, para tornar as equações mais simétricas, podemos introduzir as matrizes $\gamma$ de Dirac em que $\gamma^{0}=\beta$ e $\gamma^{i}=\beta \alpha^{i}$.

Uma representação bastante usada das matrizes $\gamma$ de Dirac é a chamada representação "padrão" (ou de Pauli) em que $\gamma^{0}$ é diagonal, ou seja,

$$
\begin{aligned}
\gamma^{0}=\left(\begin{array}{cc}
I & 0 \\
0 & -I
\end{array}\right) & \gamma^{i} \\
= & \left(\begin{array}{cc}
0 & \sigma^{i} \\
-\sigma^{i} & 0
\end{array}\right)
\end{aligned}
$$

Multiplicando a Eq. de onda ( 27 ) por $\beta$, teremos a equação de Dirac na sua forma mais usual

$$
\begin{aligned}
& \left(i \gamma^{\mu} \partial_{\mu}-m\right) \psi \\
& =0
\end{aligned}
$$

onde as matrizes $\gamma^{\mu}$ satisfazem a seguinte relação de anti-comutação:

$$
\left\{\gamma^{\mu}, \gamma^{\nu}\right\}=2 g^{\mu \nu}
$$

Usando-se a equação acima, outras relações entre as matrizes $\gamma$ tais como

$$
\gamma_{0} \gamma_{\mu}^{\dagger} \gamma_{0}=\gamma_{\mu}
$$

pode ser facilmente obtida. 
No formalismo de Dirac ainda encontramos o espinor adjunto, $\bar{\psi}=\psi^{\dagger} \gamma_{0}$ o qual satisfaz a equação de Dirac adjunta

$$
i \partial^{\mu} \bar{\psi} \gamma_{\mu}+m \bar{\psi}=0
$$

Ambas as equações, de Klein-Gordor (26) e a de Dirac (29) são equações relativísticas e descrevem, respectivamente, partículas de spin-0 e de spin-1/2. Outras equações no contexto relativístico descrevem o movimento de partículas massivas com spin maiores que $1 / 2$, tais como a equação de Proca, spin-1 e a de Rarita-Schwinger, spin -3/2. A teoria de Dirac prevê a existência de antipartículas. Segundo Dirac deve existir uma partícula com mesma massa que a do elétron, porém, com carga negativa. Tal partícula é chamada de pósitron. Apesar do sucesso, a equação de Dirac descreve somente férmions, partículas com spin semi-inteiro; ela não descreve bósons. Fenômenos, tais como produção de pares, criação ou aniquilação de partículas, efeito fotoelétrico, decaimento radioativo, emissão espontânea, etc., não são esclarecidos pela $M Q$, seja ela não-relativística ou relativística. A descrição de tais processos físicos se encontra desenvolvida no formalismo da teoria quântica de campos.
1.6 Mecânica quântica relativística nãocomutativa.

A equação de Dirac que descreve partículas relativísticas de spin $1 / 2$ no espaçotempo NCé

$$
\left[i \gamma^{\mu}\left(\partial_{\mu}-i e A_{\mu}\right)-m\right] \star \psi=0
$$

envolvendo o produto Moyal, definido pela Eq. (16), o qual não atua no termo cinético e de massa, (FERRARI, 2013), (detalhes do potencial $A_{\mu}$ serão descritos na seção 2.3 a seguir). Como uma teoria com espinores de 2 componentes em 3-D viola paridade, podemos escolher trabalhar com partículas de spin $s=$ $+1 / 2$ ou $s=-1 / 2$. As matrizes de Dirac podem ser escritas em termos das matrizes de Pauli

$$
\left(\gamma^{0}, \gamma^{1}, \gamma^{2}\right)=\left(\sigma_{3}, i \sigma_{1}, i \sigma_{2}\right)
$$

e dessa forma a Hamiltoniana total, $H$, poderá ser escrita como $H=H_{0}+H_{\text {int }}$ em que

$$
H_{0}=-i \gamma^{0} \gamma^{\ell} \partial_{\ell}+m \gamma^{0}
$$

Dependendo da forma da Hamiltoniana de interação, $H_{\text {int }}$, nos diversos potenciais de interesse, poderá ser empregado o método de teoria de perturbação. Este mecanismo permite o cálculo da $1^{a}$. e $2^{a}$. correção para a energia. Outros métodos, tais como a expansão $1 / N$, permitem ainda a resolução da equação de Dirac NC. 
1.7 O tensor do campo eletromagnético NC

O quadri-potencial do campo eletromagnético é dado por (KAKU,1993:100):

$$
\begin{aligned}
A^{\mu}=(\varphi, \vec{A})= & \left\{A_{0}, \boldsymbol{A}\right\} \\
& =\left\{A_{0}, A_{x}, A_{y}, A_{z}\right\} \\
& =g^{\mu \nu} A_{v}
\end{aligned}
$$

em que as componentes contravariantes do $A^{\mu}$ estão explicitamente descritas; $A_{\mu}=$ $\left\{A_{0},-A_{x},-A_{y},-A_{z}\right\}$ são as componentes covariantes deste quadri-vetor. A partir de $A^{\mu}$ o tensor do campo eletromagnético pode ser escrito como:

$$
\begin{aligned}
F^{\mu \nu}=\frac{\partial A^{\mu}}{\partial x_{v}}- & \frac{\partial A^{v}}{\partial x_{\mu}} \\
& =\left(\begin{array}{cccc}
0 & E_{1} & E_{2} & E_{3} \\
-E_{1} & 0 & B_{3} & -B_{2} \\
-E_{2} & -B_{3} & 0 & B_{1} \\
-E_{3} & B_{2} & -B_{1} & 0
\end{array}\right),
\end{aligned}
$$

o qual é um tensor anti-simétrico de segunda ordem.

As equações de Maxwell $\operatorname{div} \vec{E}=\rho$, $\operatorname{rot} \vec{B}=\partial \vec{E} / \partial t+\vec{\jmath}$ são equivalentes a $\partial_{\mu} F^{\mu v}=j^{v}$

neste $j^{v}=(\rho, \vec{\jmath})$ é o quadrivetor densidade de corrente. Para as equações de Maxwell homogêneas o $\operatorname{rot} \vec{E}=-\partial \vec{B} / \partial t$ e $\operatorname{div} \vec{B}=0$ são escritos na forma covariante como:

$\partial_{\mu} \tilde{F}^{\mu \nu}=0$ em que o tensor $\tilde{F}^{\mu \nu}$ é o dual $F^{\mu \nu}$ e a relação entre os mesmos é

$\tilde{F}^{\mu \nu}=\frac{\epsilon^{\mu \nu \rho \sigma}}{2} F_{\rho \sigma}$.

$\epsilon^{\mu \nu \rho \sigma}$ é o símbolo de Levi-Civita quadridimensional o qual vale $+1(-1)$, se $(\alpha, \beta, \gamma, \delta)$ formar uma permutação par (impar) de $(0,1,2,3)$, e vale 0 (zero) nos outros casos. A equação ( 34 ) é automaticamente satisfeita se $F^{\mu \nu}=\partial^{\mu} A^{v}-\partial^{v} A^{\mu}$.

\subsection{1 $\widehat{\boldsymbol{F}}_{\mu v}$ no plano comutativo}

Todas as quantidades com $\wedge$ (“batted" quantidades) estão imersas no espaço nãocomutativo. Neste caso, o tensor do campo eletromagnético é escrito como:

$$
\begin{aligned}
\hat{F}_{\mu \nu}=\partial_{\mu} A_{\nu}- & \partial_{\nu} A_{\mu} \\
& -i\left[A_{\mu}, A_{\nu}\right]_{\star}
\end{aligned}
$$

onde

$$
\left[A_{\mu}, A_{\nu}\right]_{\star}=A_{\mu} \star A_{\nu}-A_{\nu} \star A_{\mu}
$$

é o comutador NC conforme Eq. (12). Os termos envolvendo do produto Moyal em ( 37 ), até a aproximação linear em $\theta$, podem assim ser calculados:

$$
\begin{array}{r}
-i\left[A_{\mu}, A_{\nu}\right]_{\star}=-i\left(A_{\mu} A_{v}+\frac{i}{2} \theta^{\alpha \beta} \partial_{\alpha} A_{\mu} \partial_{\beta} A_{\nu}\right) \\
+i\left(A_{\nu} A_{\mu}+\frac{i}{2} \theta^{\beta \alpha} \partial_{\beta} A_{\nu} \partial_{\alpha} A_{\nu}\right) \\
= \\
=-i\left(\frac{i}{2} \theta \epsilon^{\alpha \beta} \partial_{\alpha} A_{\mu} \partial_{\beta} A_{v}-\frac{i}{2} \theta^{\beta \alpha} \partial_{\beta} A_{\nu} \partial_{\alpha} A_{\mu}\right)
\end{array}
$$




$$
=\theta \epsilon^{\alpha \beta} \partial_{\alpha} A_{\mu} \partial_{\beta} A_{v}
$$

Dessa forma, o tensor do campo eletromagnético torna-se:

$$
\begin{aligned}
& \hat{F}_{\mu \nu} \\
& =\partial_{\mu} A_{\nu}-\partial_{\nu} A_{\mu} \\
& +\theta \epsilon^{\beta \alpha} \partial_{\alpha} A_{\mu} \partial_{\beta} A_{\nu} .
\end{aligned}
$$

A ação de Maxwell livre em espaços nãocomutativos é dada por:

$$
\begin{aligned}
S=- & \frac{1}{4} \int d^{4} x F_{\mu \nu} \star F_{\mu \nu}=-\frac{1}{4} \int d^{4} x \hat{F}^{2}{ }_{\mu \nu}= \\
= & \int d^{4} x\left\{-\frac{1}{4} F^{\mu \nu} F_{\mu \nu}\right. \\
& -\frac{1}{4} e^{2} \theta^{4}\left(\partial^{\alpha} A^{\mu} \partial_{\alpha} A_{\mu} \partial^{\beta} A^{v} \partial_{\beta} A_{\nu}\right. \\
& \left.\left.-\partial^{\alpha} A^{\mu} \partial_{\alpha} A_{\nu} \partial^{\beta} A^{v} \partial_{\beta} A_{\mu}\right)\right\} .
\end{aligned}
$$

A ação dada pela acima é invariante com respeito às transformações de Lorentz (estão escritas na forma covariante); é ainda $\mathcal{C}_{-}, \mathcal{P}$ - e $\mathcal{T}$-invariante. As correções para a não-comutatividade são da $\mathcal{O}\left(\theta^{4}\right)$.

4. Efeitos Da Não-Comutatividade Canônica

Apresentaremos a seguir uma aplicação deste formalismo na mecânica quântica nãorelativística em que o parâmetro não-comutativo $\theta$ é constante (GOUBA,2016;2), ou seja, empregaremos a não-comutatividade canônica. Nesta situação a não comutatividade do espaço é realizada pelos operadores coordenadas satisfazendo a equação

$$
\left[\hat{x}_{i}, \hat{x}_{j}\right]=i \theta_{i j}
$$

onde os operadores $\hat{x}_{i}, i=1,2$ são operadores coordenadas e $\theta_{i j}=\theta \epsilon_{i j}$, em que $\epsilon_{i j}$ é o tensor 2- $D$ totalmente antissimétrico $\left(\epsilon_{12}=1\right) \mathrm{e}$ $\epsilon_{j k} \epsilon_{k \ell}=-\delta_{j \ell} \operatorname{com} \theta$ uma constante.

4.1 O oscilador harmônico 2-D nãocomutativo

Recentemente, em (CHUNG, 2016), o oscilador harmônico 2-dimensional NC foi considerado no contexto tanto da nãocomutatividade do espaço como do momento e em álgebras não-comutativas mais gerais.

Vamos considerar o oscilador harmônico NC de massa $M$ e frequência $\omega$ em 2D. A dinâmica do sistema, conforme modificação dada pela Eq. ( 20 ), é dada pelo operador Hamiltoniano

$$
\begin{aligned}
H=\frac{1}{2 M}\left[p_{j} p_{j}\right. & +M^{2} \omega^{2}\left(x_{j}-\frac{\theta}{2 \hbar} \epsilon_{j k} p_{k}\right)\left(x_{j}\right. \\
& \left.\left.-\frac{\theta}{2 \hbar} \epsilon_{j \ell} p_{\ell}\right)\right] \\
& \equiv(1 \\
& \left.+\frac{M \omega^{2} \theta^{2}}{4 \hbar^{2}}\right) H_{\theta}
\end{aligned}
$$

onde 
$H_{\theta}$

$=\frac{1}{2 M}\left[p_{j} p_{j}+M^{2} \omega_{\theta}^{2} x_{j} x_{j}\right.$

$\left.-M^{2} \omega_{\theta}^{2} \theta \epsilon_{j k} x_{j} p_{k}\right]$

e

$$
\omega_{\theta}^{2} \equiv \frac{\omega^{2}}{\left(1+\frac{M \omega^{2} \theta^{2}}{4}\right)}
$$

Os operadores de criação $a_{j}^{\dagger}$ e de aniquilação $a_{j}$ obedecem as relações de comutação:

$$
\begin{aligned}
& {\left[a_{j}, a_{k}\right]=0} \\
& {\left[a_{j}^{\dagger}, a_{k}^{\dagger}\right]=0}
\end{aligned}
$$

$\left[a_{j}, a_{k}^{\dagger}\right]=\delta_{j k}$,

que em termos de $x_{j}$ e de $p_{j}$ podem ser escritos como:

$$
\begin{gathered}
x_{j} \\
=\sqrt{\frac{\hbar}{2 M \omega_{\theta}}}\left(a_{j}^{\dagger}\right. \\
\left.+a_{j}\right) \\
p_{j}=i \sqrt{\frac{\hbar M \omega_{\theta}}{2}\left(a_{j}^{\dagger}\right.} \\
\left.-a_{j}\right)
\end{gathered}
$$

O espectro de energia do oscilador harmônico 2-D no caso comutativo é dado por

$$
\begin{aligned}
E_{n, m}=E_{n}+ & E_{m}=(n+m+1) \hbar \omega \\
& =(N \\
+ & 1) \hbar \omega
\end{aligned}
$$

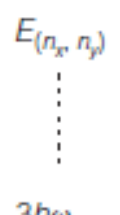

$3 h \omega$

$2 h \omega$

$h \omega$

$(E=0)$ $\left(n_{x}, n_{y}\right)$

$(2,0),(1,1),(0,2)$

$(1,0),(0,1)$
Fig. 1 - Espectro da energia e degenerescências para o oscilador harmônico 2-D comutativo em coordenadas cartesianas.

A substituição das equações (43) e ( 44 ) em ( 42 ) resulta

$$
\begin{aligned}
H_{\theta}=\hbar \omega_{\theta}[N & +I \\
& -\frac{4 \theta}{\hbar} M \omega_{\theta} \frac{1}{2 i}\left(a_{1}^{\dagger} a_{2}\right. \\
& \left.\left.-a_{2}^{\dagger} a_{1}\right)\right]
\end{aligned}
$$

onde $I$ é o operador identidade. Para o momento angular na representação de Schwinger, temos:

$$
\begin{gathered}
J_{1}=\frac{1}{2}\left(a_{2}^{\dagger} a_{1}-a_{1}^{\dagger} a_{2}\right) \\
J_{2}=-\frac{i}{2} a_{j}^{\dagger} \epsilon_{j k} a_{j} \\
=\frac{1}{2 i}\left(a_{2}^{\dagger} a_{1}-a_{1}^{\dagger} a_{2}\right)
\end{gathered}
$$

$$
J_{3}=\frac{1}{2}\left(a_{1}^{\dagger} a_{1}-a_{2}^{\dagger} a_{2}\right)
$$

$$
J^{2}=J_{k} J_{k}=\frac{N}{2}\left(\frac{N}{2}+1\right)
$$


Dessa forma, a parte $a_{1}^{\dagger} a_{2}-a_{2}^{\dagger} a_{1}$ na Eq. (46) pode ser identificada com $2 i J_{2}$. Porém, sob uma transformação unitária

$$
\left(\begin{array}{l}
a_{1} \\
a_{2}
\end{array}\right)=\frac{1}{\sqrt{2}}\left(\begin{array}{ll}
1 & -i \\
i & -1
\end{array}\right)\left(\begin{array}{l}
a_{1}^{\prime} \\
a_{2}^{\prime}
\end{array}\right)
$$

esta torna-se $2 i J^{\prime}{ }_{3}$. Com esta mudança para a Hamiltoniana $H_{\theta}$ em (46) teremos um problema de autovalores da energia

$$
H_{\theta}|j m\rangle=\hbar \omega_{\theta}\left(n+1-2 \theta m M \omega_{\theta}\right)|j m\rangle
$$

em que $J^{\prime}{ }_{3}|j m\rangle=m \hbar|j m\rangle$ e $|j m\rangle$ são os autovetores de $J^{2}$ e $J_{2}$. Tais autovetores são rotulados pelos autovalores de $J^{2}$ e $J_{2}$, ou seja, respectivamente por $j$ e $m$.

$$
j=0, \frac{1}{2}, 1, \frac{3}{2}, 2, \ldots
$$

Os $m$-valores para um dado $j$ são

$$
m=\underbrace{-j, j+1, \ldots, j-1, j}_{2 j+1 \text { estados }}
$$

e

$n=2 j$.

$\mathrm{Na}$ situação comutativa $(\theta=0)$ a

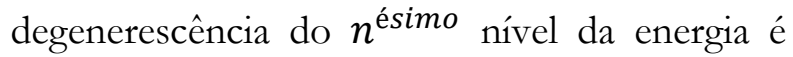
$2 j+1=n+1$. Dessa forma, a degenerescência é levantada pela nãocomutatividade, i.e., os níveis não terão mais a mesma energia.

Outros efeitos dessa natureza, similarmente surgem em física. O efeito Stark (LONGAIR, 2013:146), o efeito Zeeman
(ATKINS;FRIEDMAN,2005:248), bem como a rede de translação ("lattice translation"), a qual é um tipo de operação de simetria discreta com importantes aplicações em física do estado sólido (SAKURAI, 1994:280), são alguns exemplos encontrados na literatura.

\section{Considerações Finais}

Neste trabalho foi apresentada a mecânica quântica não-comutativa e destacados alguns de seus essenciais aspectos tanto na situação relativística quanto não-relativística. Empregamos em ambas as situações a chamada não-comutatividade canônica, i.e., aquela em que o parâmetro não-comutativo $\theta$ é constante. $\mathrm{Na}$ situação relativística, assumindo que os efeitos da não-comutatividade são pequenos, mostramos como se transforma o tensor do campo eletromagnético expandido o produto Moyal e retendo somente os termos lineares em $\theta$. Com tais implementos temos que ação é invariante com respeito às transformações de Lorentz, bem como, por conjugação de carga, paridade e inversão temporal. A seguir, concluímos com uma aplicação desse formalismo no estudo do oscilador harmônico NC em duas dimensões na situação nãorelativística. Usando o método dos operadores de Dirac constatamos que o espectro da energia é degenerado e sua degenerescência é levantada pela não-comutatividade. 
Nesta linha, surgiram diversos trabalhos para a não-comutatividade, tais como, a dependente da posição (KUPRYIANOV, GOMES, 2008) e a envolvendo uma mistura de graus de liberdade espacial e de spin (FERRARI, 2013). Muitas questões, no entanto, se encontram ainda em aberto e outros tipos de potenciais podem ser investigados. Uma questão ainda de particular interesse são os limites fenomenológicos do parâmetro $\theta$ da nãocomutatividade. Estudos indicam que este é da ordem de $\sim(10 \mathrm{Tev})^{-2}$, o que denota que os efeitos da não-comutatividade estão no campo da física de alta energia.

\section{Referências Bibliográficas E Eletrônicas}

ATKINS, P; FRIEDMAN, R. Molecular Quantum Mechanics, 5a. ed., Oxford University Press, 2005. Disponível em: https://books.google.com.br/books?id=9k-

$$
\text { cAQAAQBAJ }
$$
\&printsec $=$ $\underline{\text { frontcover\&dq }=\text { atkins }+ \text { molecular }+ \text { quantum }+}$ $\underline{\text { mechanics\&hl }=p t B R \& s a=X \& v e d=0 \text { ahUKEwj }}$ b9OeLz7bNAhVDGpAKHdlJAm8Q6wEIHT $\underline{\mathrm{AA} \# \mathrm{v}=\text { onepage } \& \mathrm{q}=\text { zeeman } \& \mathrm{f}=\text { false }}$

BASSALO, J. M. F. Eletrodinâmica Quântica, $1^{\text {a }}$. ed., Editora Livraria da Física, 2006.

CHUNG, W. S. Two-dimensional noncommutative quantum mechanics with the central potential. Disponível em: http://www.worldscientific.com/doi/10.1142/ $\underline{\mathrm{S} 0217732316500462}$
FERRARI, A.; GOMES, M.; KUPRIYANOV, V. G.; STECHHAHN, C. A. 1/N expansion in noncommutative quantum mechanics. Phys. Rev. D 82 (2010). Disponível em: 10.1103/Phys $\underline{\text { RevD.82.045009 }}$

FERRARI, A.; GOMES, M.; V. G. KUPRIYANOV; STECHHAHN, C. A. Dynamics of a Dirac férmion in the presence of spin noncommutativity. Physics Letters B 718 (2013) 1475-1480. Disponível em: http://dx.doi.org/10.1016/i.physletb.2012.12.0 $\underline{10}$

GASIOROWICZ, S. Quantum physics, 3ª ed. John Wiley \& Sons, Inc, 2003.

GIROTTI, H. O. Noncommutative quantum field theories. Disponível em: https://arxiv.org/ pdf/hep-th/0301237.pdf

GOLDSTEIN, H. Classical Mechanics, $2^{\mathrm{a}}$. ed. Addison-Wesley Pub. Company, 1980.

GOMES, M. Teoria Quântica de Campos, $1^{\mathrm{a}}$. ed., Edusp, 2002.

GOUBA, L. Four formulations of noncommutative quantum mechanics. Disponível em: $\quad \underline{\text { http:// }}$ arxiv.org/pdf/1603.07176.pdf

GREINER, W. Relativistic Quantum Mechanics - Wave equations, Springer, 2000.

GRIFFITHS, D. J. Introduction to Quantum Mechanics, $2^{\mathrm{a}}$. ed., Pearson Prentice Hall, 2005. 
KAKU, M. Quantum Field Theory, 1ª ed. Oxford University Press, 1993.

KUPRIYANOV, V. G. Quantum mechanics with coordinate dependent noncommutativity. Disponível em: http://arxiv.org/pdf/1204.4823.pdf

LONGAIR, M. Quantum Concepts in Physics, $1^{\text {a }}$. ed., Cambridge, 2013.

OLIVEIRA, Denny Mauricio de. Uma proposta para o ensino de teoria quântica de campos na graduação: a eletrodinâmica de Maxwell-ChernSimons como motivação. Revista Brasileira de Ensino de Física, v. 33, n. 3, 3309 (2011). Disponível em:

http://www.scielo.br/scielo.php?

$\underline{\text { Script }}=$ sci abstract\&pid $=$ S1806-

$11172011000300009 \& \ln g=$ en\&nrm $=$ iso\&tlng $=$ $\underline{\text { en }}$

RINDLER, W. Relativity Special, General and Cosmological, $2^{\mathrm{a}}$. ed., Oxford, 2006.

SAKURAI, J. J.; Napolitano, J. Modern Quantum Mechanics, 2a. ed., Addison-Wesley, 1994.

SEIBERG, N.; WITTEN E., JHEP 9909, 032 (1999).

Disponível em:

https://arxiv.org/pdf/hep-th/9908142v3.pdf

STECHHAHN, C. A. Efeito Aharonov-Bohm não-comutativo para partículas relativísticas de spin $1 / 2$. Tese (2005). Universidade de São Paulo.

Disponível em:

\section{http://www.teses.usp.br/}

teses/disponiveis/43/43134/tde-02042007-

\section{4/pt-br.php}

